\title{
DHI Analysis Using Seismic Frequency Attribute On Field-AN Niger Delta, Nigeria
}

\author{
Yaqub O. Adepoju ${ }^{1}$, Joseph O. Ebeniro ${ }^{2}$, Chukwuemeka N. Ehirim ${ }^{3}$ \\ ${ }^{I}$ (Physics Department, University of Port Harcourt, Nigeria) ${ }^{2}$ (Physics Department, University of Port Harcourt, \\ Nigeria) $)^{3}$ (Physics Department, University of Port Harcourt, Nigeria)
}

\begin{abstract}
Effect of hydrocarbon gas on the seismic frequency attributes was studied and considered for applicability as Direct Hydrocarbon indicator (DHI) in the AN reservoir in the Niger Delta basin. Matching Pursuit algorithm for the spectral decomposition was adopted for frequency attributes analysis at $10 \mathrm{~Hz}$ to $35 \mathrm{~Hz}$, along the interpreted reservoir top. Three frequency surface attributes were co-rendering and color blending to highlight the effect of gas in the reservoir. Gas filled sand exhibits distinctly higher frequency amplitudes at low frequency bands of $15 \mathrm{~Hz}$ to $25 \mathrm{~Hz}$ despite a subtle hardness of the sand relative to the overburden thick shale. High frequency amplitude expression in the low frequency $(15 \mathrm{~Hz}-25 \mathrm{~Hz})$ band observed in the gas reservoir is found to be a consistent character of overpressured gas reservoirs within the Niger Delta basin. It was concluded, therefore based on time-frequency seismic character of the gas sand, that frequency attribute can be used as a direct hydrocarbon indicator for the sandstone gas reservoir in the Niger Delta.
\end{abstract}

Keywords: Seismic attribute, Spectral Decomposition, Niger delta

\section{Introduction}

Within the Eastern Ughelli depo-belt of the Niger delta lays the Field-AN with stacked reservoirs laden with high GOR hydrocarbon resources. We refer to a particular R1 reservoir which is penetrated by six (6) wells, three (3) of which logged gas-down-to (GDT), while two well near the flanks logged gas water contact (GWC) and oil water contact (OWC). A well at the edge of the field however logged only water. In a $70 \mathrm{~km} 2$ field with $40 \%$ of this area being geologically map-able hydrocarbon contact, a seismic amplitude support would be considered desirable as a key supporting evidence for establishing field wide contact certainty for reserve estimate. This is however not the case because the traditional seismic reflection amplitude extraction from full stack volume Pre-stack Depth Migration seismic data along R1 reservoir sand top is patchy and inconsistent across the field. It therefore cannot be applied to support even a pessimistic hydrocarbon resource estimate.The patchy amplitude map (Fig. 1) was derived by root mean square (RMS) amplitude extraction within a window of 20miliseconds around the interpreted R1 sand top. This presents a geophysical challenge for seismic interpreters to solve. Whenever this challenge becomes unresolvable, it usually leaves the geologists to depend only on structural map and contacts information logged from the wells that penetrate such reservoirs. It also usually backed with an assumption of good sand development across the field. This type of geological deductions results in an estimate that will not be free of significant uncertainties. The condition becomes more challenges in this case where only two well logged hydrocarbon contacts.

Indeed, there are many variables that can create multiple geological scenarios which lead to wider uncertainties about sand development in the field. These variables include the presence of intra-reservoir shales baffles, intra-reservoir faults and lateral facie changes that create porosity variation within the reservoir.

Spectral decomposition is applied in imaging and mapping of bed thickness and geologic discontinuities [1]. This was achieved by transforming the seismic data from time-reflectivity amplitude domain into the timefrequency domain with the discrete Fourier transform. With this technology, seismic interpreters can quickly and effectively quantify thin bed interference and detect subtle discontinuities within large 3D surveys. Spectral decomposition was also applied as a tool for isolating the reflectivity of hydrocarbons in a Frio sand reservoir using migrated data [2]. Synthetic seismic forward modeling was modeled to explain the low-frequency hydrocarbon anomaly observed from the spectral decomposition of the far-angle stack seismic data from a deepwater West Africa reservoir [3]. Attenuation, fluid type, reservoir thickness and stratigraphy are part of the four controlling factors that were identified from that study.

Our objective therefore is to reduce the contact uncertainty especially in the static realm by applying geophysical processes within seismic interpretation workspace to provide support for hydrocarbon resource estimate and field development plan. We will generate structural conforming seismic anomaly attribute map which will consistently define the hydrocarbon contact along this huge reservoir.

The R1 reservoir studied has an average reservoir thickness of about 250ft and hydrocarbon gas being the dominant fluid. Therefore, this study narrows down the search for an explanation of the patchy amplitude map 
to two significant factors which are the reflectivity series and the fluid type. The reflectivity series is dependent on the rock properties which can be derived from the well acoustic logs.

\section{Spectral Decomposition Methods}

Where the standard seismic reflectivity amplitude maps do not provide support for a structural map, there is an available option within the seismic frequency domain to produce an attribute map that can match the geological scenario presented in the top structure map. Spectral decomposition refers to any method that produces a continuous time-frequency analysis of a seismic trace. Thus, a frequency spectrum is output for each time sample of the seismic trace [4]. Most of the early work has utilized the Short Time Fourier Transform (STFT) in which time-frequency spectrum is produced by taking the Fourier transform over a chosen time window. This process depends on the user specified window length and thus with limited resolution.

Continuous Wavelet Transform (CWT) is used to divide a continuous-time function into wavelets. Unlike Fourier transform, the CWT possesses the ability to construct a time-frequency representation of a signal that offers very good time and frequency localization. Continuous wavelet transform decomposition techniques has a place in interpretation seismology for spectral decomposition and has an advantage over STFT in that the window implicit in the wavelet used is frequency dependent. CWT produces an improved resolution for a nonstationary signal in addition to the avoidance window length specification required for the STFT [5].

Matching pursuit (MP) introduced by Mallat and Zhang [6] is a type of numerical technique which involves finding the "best matching" projections of multidimensional data on a highly redundant dictionary of functions. It is described by the equations

$$
f(t)=\sum_{n=0}^{+\infty} \alpha_{n} g_{y n}(t)
$$

where, for an input signal $f(t)$ and any dictionary, list of coefficients $\alpha_{n}$ (index function) and $g_{\gamma n}$ (sum of scalar functions called atoms) are sorted as sub-optimal solution to the problem of sparse signal representation. MP spectral decomposition technique is a computationally intensive process than the CWT and STFT. It has superior spectral resolution if a compact mother wavelet is utilized.

\section{Methods \& Materials.}

Seismic and well data as well as computer applications utilized for subsurface data interpretation were supplied and approved by SPDC Limited, Port Harcourt. Zero phased Pre-Stack Depth Migration seismic (PSDM) with 180o polarity was the input reflectivity volume for spectral decomposition process. Well to seismic tie and synthetic generation pseudo forward modeling was also carried out as a diagnostic prior to spectral decomposition work.

PreStack Depth Migration seismic was calibrated using well logs. During the well to seismic tie analysis, the dominant frequency about which most geologic features in study area are expected to be seismically embedded was determined. A comparison of the three algorithms was made by subjecting a seismic trace along inline 7500 and cross-line 15800 to spectral decomposition using the three algorithms and the comparison of the outputs is presented (Fig. 2).

On the strength of better reflector resolution, the field seismic data was subjected to matching pursuit's spectral decomposition method. The algorithm was adopted with consideration for 100 atoms with residual threshold of 0.02 knowing that the widest trace window within the synthetic 3D volume will be about $400 \mathrm{~ms}$. A seismic frequency spectrum of between $10 \mathrm{~Hz}$ to $35 \mathrm{~Hz}$ was observed from the wavelet extracted from the $3 \mathrm{D}$ seismic volumes and that formed the basis for setting a frequency bandwidth of interest.

Also, for the purpose of output comparison, the following spectral decomposition configuration setting for the STFT and CWT were also applied. Short Time Fourier Transform (STFT) with windows length of $120 \mathrm{~ms}$ with $20 \%$ of the windows length as taper ends. While $20 \mathrm{~Hz}$ and $25 \mathrm{~Hz}$ frequency bands were targeted because the dominant frequency is observed to be about $24 \mathrm{~Hz}$. Manual amplitude clipping ranged between -100 and 100000 to capture all ranges of frequency amplitude that may give indications of geology, rock characteristics and fluid content. Continuous Wavelet Transform (CWT) algorithm with a Ricker wavelet was run for $20 \mathrm{~Hz}$ and $25 \mathrm{~Hz}$ frequency bands with manual amplitude clipping range of between -100 and 100000

\section{Results \& Discussion}

As predicted by the diagnostics (Fig. 2), the comparison of the three spectral decomposition methods suggested that the matching pursuit was able to resolve the seismic reflector better than the Short Time Fourier Transform (STFT) and the Continuous Wavelet Transform (CWT). Critical requirements for successful spectral decomposition process include the computing power and data storage spaces especially if a wide frequency 
range is being processed. The output of the $10 \mathrm{~Hz}$ to $35 \mathrm{~Hz}$ spectrum with $5 \mathrm{~Hz}$ stepping spectral decomposition exhibits variation in the frequency amplitude across this spectral range.

The full stack PSDM seismic volume in time-frequency domain using the Matching Pursuit is observed to express lot more details of the subsurface structures as well as stratification that maps closely with the gamma ray log signatures. High amplitude expression map sands on the well log while the dark coloured low amplitude frequency expression map shale section of the log. These observations were made on all the six (6) frequency volumes from $10 \mathrm{~Hz}$ to $35 \mathrm{~Hz}$ volume (Fig. 3).

It is also observed that the best amplitude expression is on the frequency volume around the dominant frequency. The amplitude shut off is sharp and conforms to the structure mapped from the reflectivity seismic volume. Seismic frequency attributes extracted along the top reservoir coincides with the high amplitude low frequency volumes of $15 \mathrm{~Hz}, 20 \mathrm{~Hz}$ and $25 \mathrm{~Hz}$. The three attribute maps were co-rendered with blending options iteratively until a combination that best expressed the gas accumulation was obtained.

Some part of the reservoir exhibit low amplitude expression even at low frequency (Fig. 4). Much of the gas zone however expresses high frequency amplitude at a low frequency band of between $15 \mathrm{~Hz}$ and $25 \mathrm{~Hz}$. An area with reduced expression of gas presence coincides with the location of the AS-01 well, thus providing an opportunity to analyze the well for clues. The analysis of the rock properties indicates subtle acoustic impedance difference between this reservoir rock and the overburden shale (Fig. 5). The overburden shale which is overpressured by $0.18 \mathrm{psi} / \mathrm{ft}$, is acoustically slower than the gas sand but the shale density remain fairly constant in spite of the velocity reversal (Fig. 6). Low acoustic impedance contrast also translates to absence of low frequency response at these parts of the shale-sand interface. The lateral improvement in the acoustic impedance contrast at the reservoir top explains the high amplitude expression toward the east.

\section{Figures}

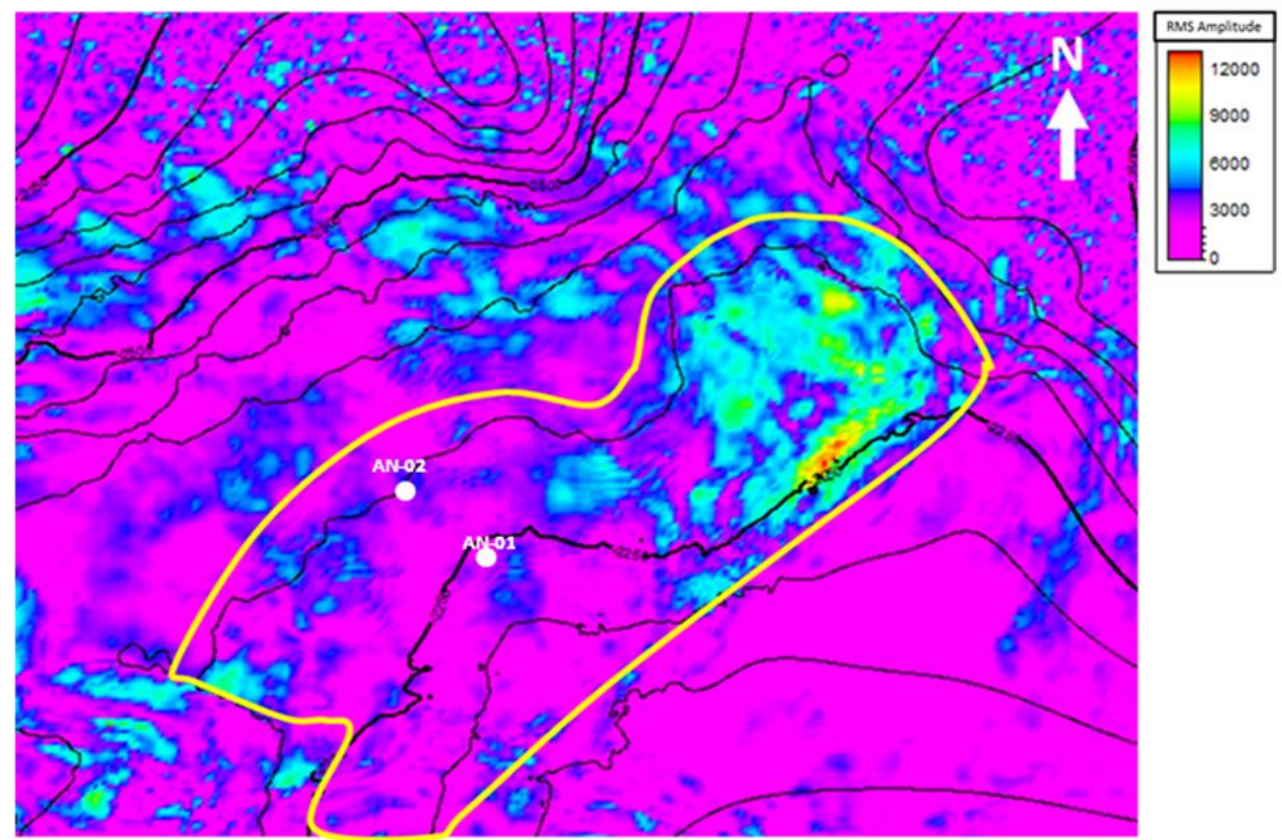

Fig. 1: Reflection amplitude map of Field-AN R1 reservoir top. It shows a patchy amplitude anomaly which lacks geological inconsistency with the structural map. At AN-01 well location and it surrounding, low seismic reflection amplitude is expressed due to poor resolution of the sand-shale interface. At the northeast part of the field, amplitude expression improved. This is a result of higher acoustic impedance contrast at the sand-shale interface. AN-01 logged a Gas-Down-To (GDT) while AN-02 logged both a Gas-Water-Contact (GWC) and Oil-Water-Contact (OWC). 

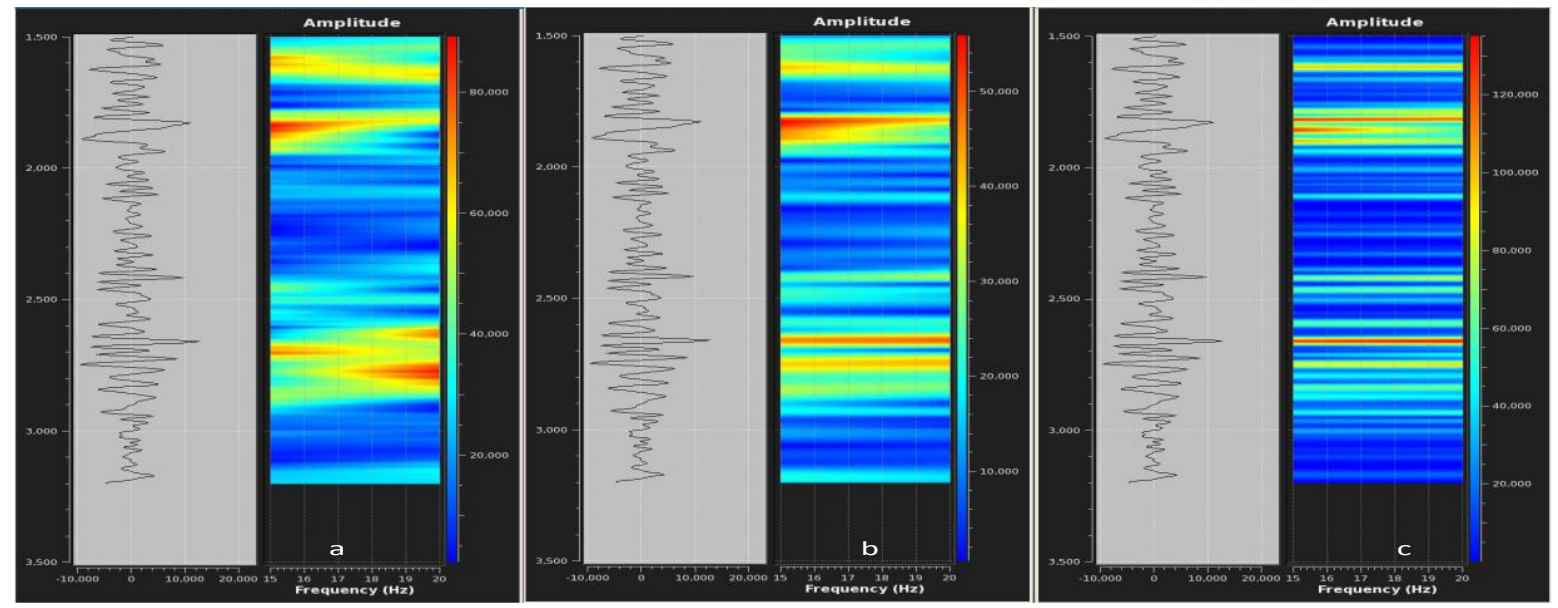

Fig. 2: A higher resolution sample output from the Matching Pursuits algorithm (c.) while sample output from the Continuous Wavelet Transform (b) and Short Time Frequency Transform (a) algorithms are smeary.
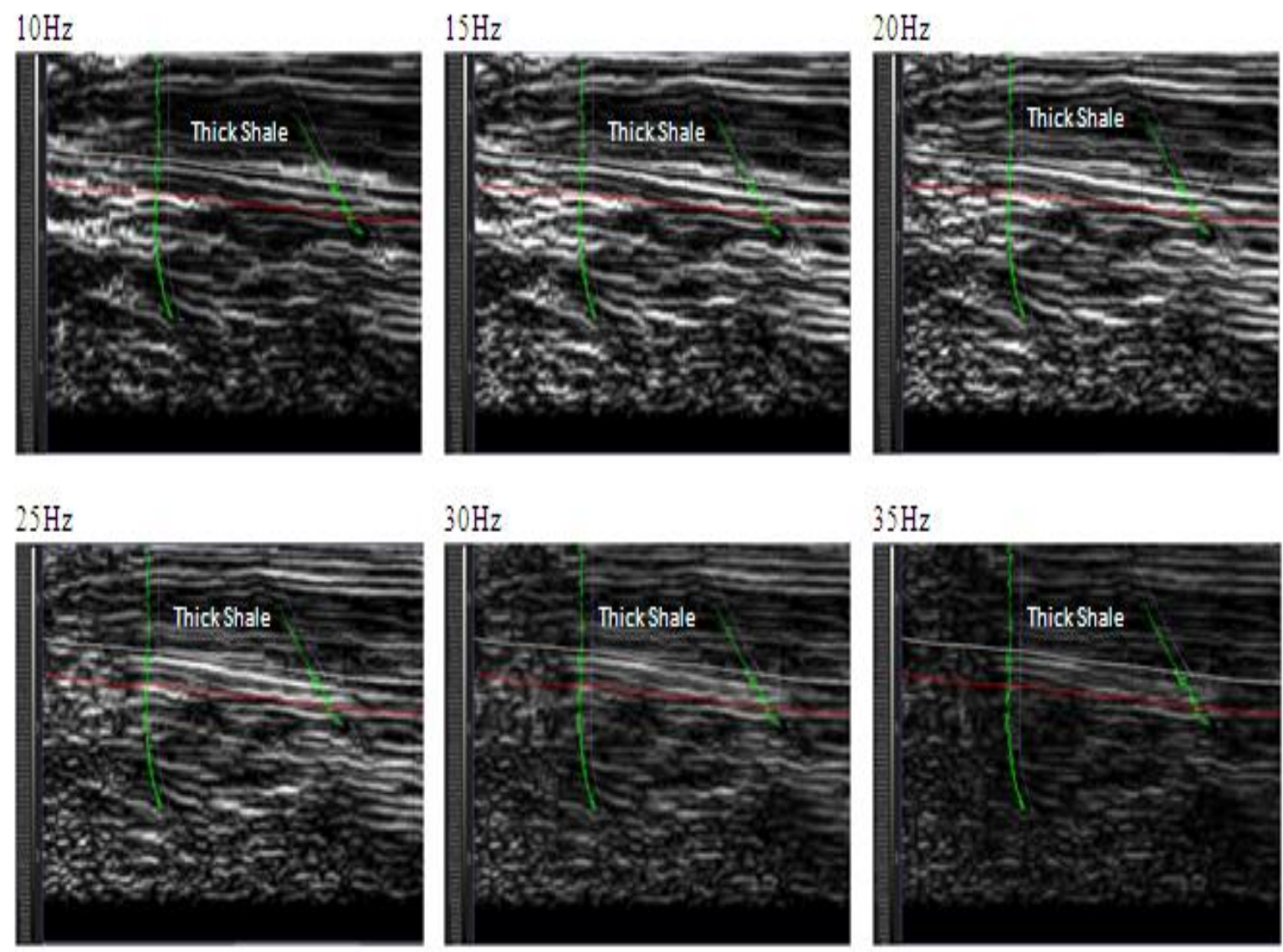

Fig. 3: Trace sections showing the low frequency volumes with high amplitude coinciding with the sand reservoirs calibrated with gamma-ray logs. The vertical well is the AN-01 while the deviated well is the AN-02. The grey scale is representative of the amplitude scale, white being high while dark being low. At $25 \mathrm{~Hz}$, the brightness along the interpreted surface (red line) connects the two wells just at the gas contacts logged in these wells. The thick shale unit overlying the sand is particularly visible as dark grey section overlying the bright gas sand unit. 

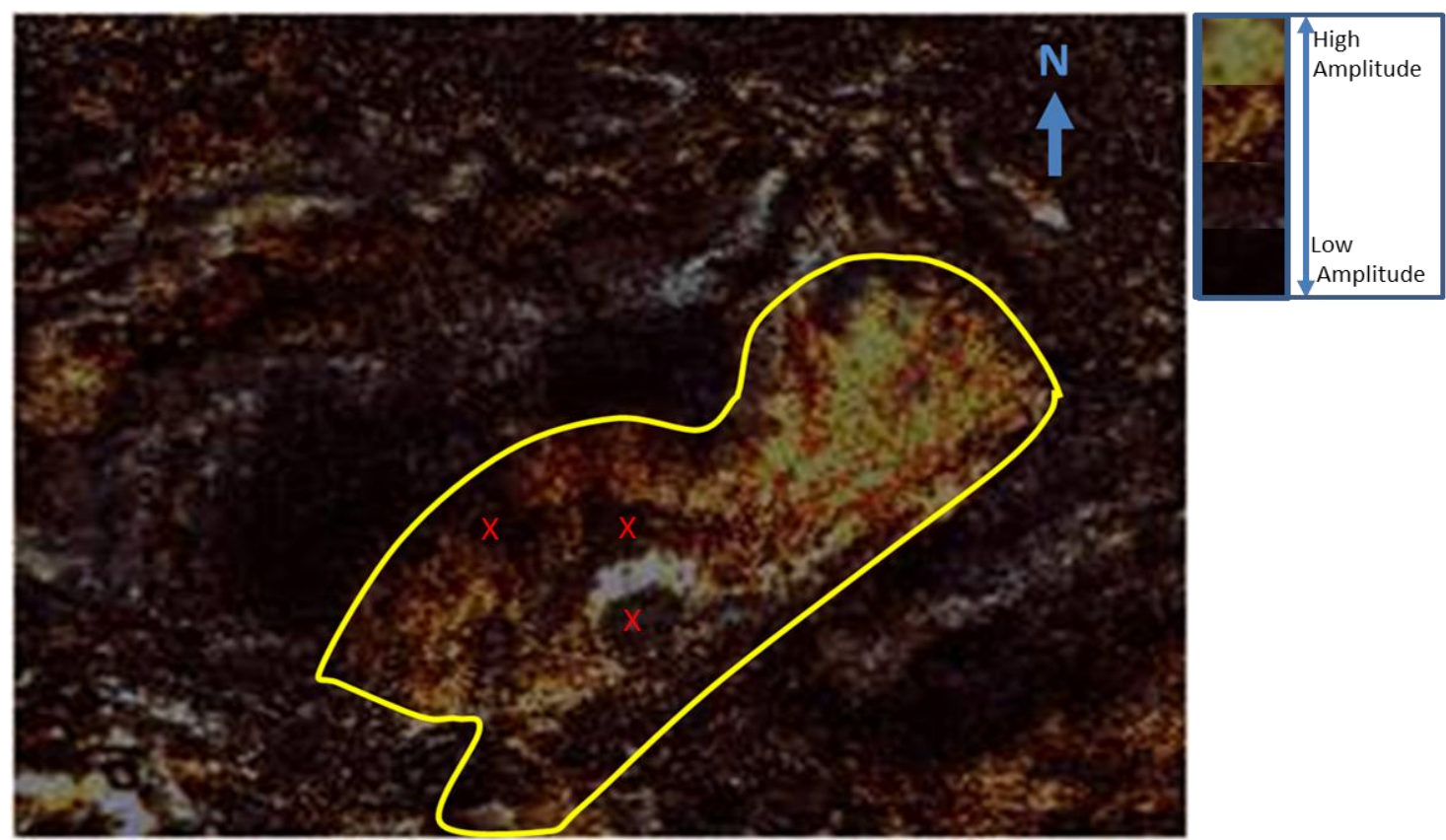

Fig. 4: Co-rendered overlays of the frequency amplitude map of $15 \mathrm{~Hz}, 20 \mathrm{~Hz}$ and $25 \mathrm{~Hz}$ spectra. This output better defines the gas contact, which is conformable to the mapped structure. Areas with low amplitude expression within the low frequency band data marked $\mathrm{X}$, corresponds to area with smallest acoustic impedance contrast between the overburden shale and the gas sand.

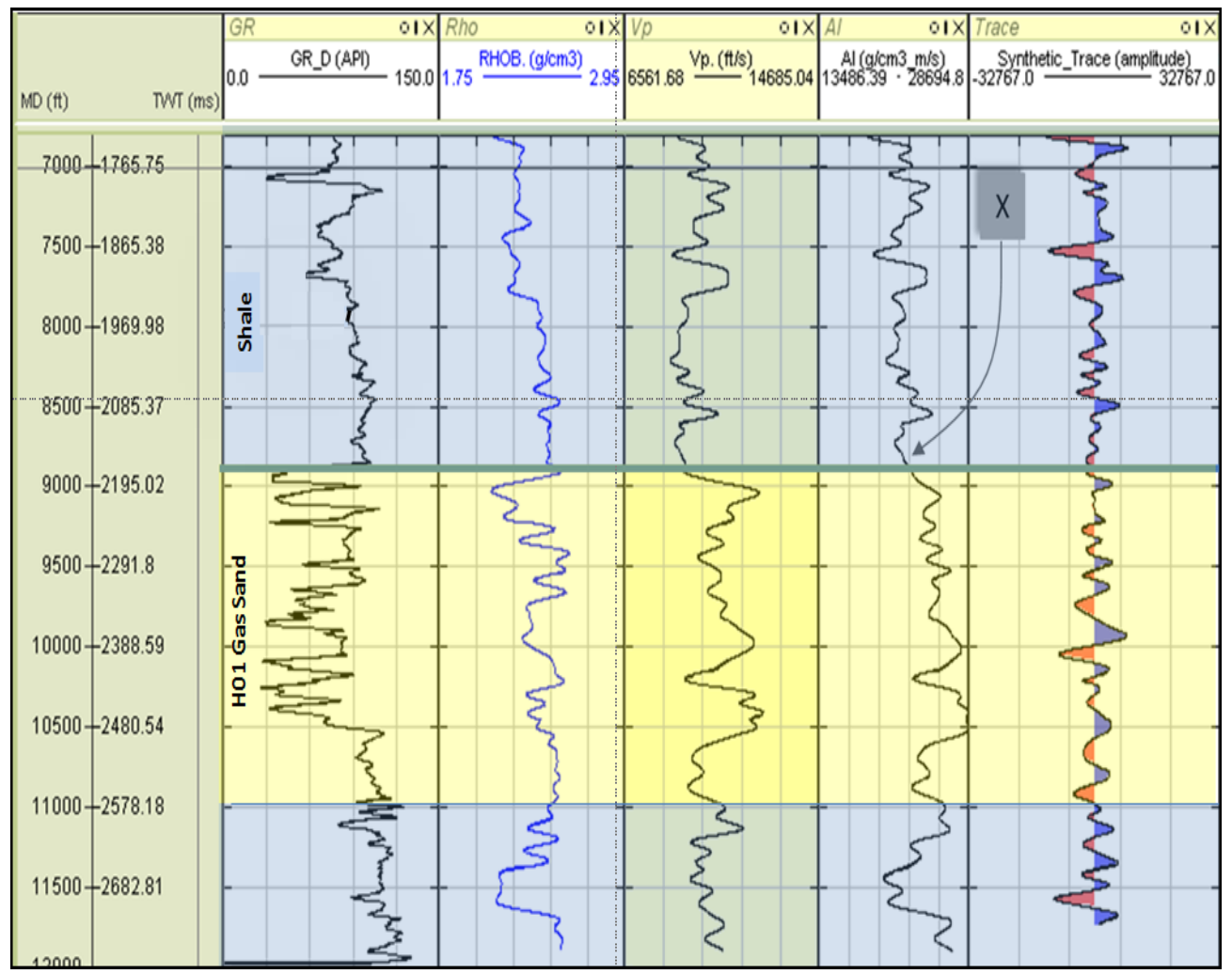

Fig. 5: A $\log$ panel view of $\mathrm{AN}-01$ well. Point $\mathrm{X}$ along the acoustic impedance log highlights the subtle contrast at the interface between soft thick shale and the R1 gas sand. This results in high frequency, low amplitude response of the synthetic seismic trace as well as the field seismic traces at the shale-gas sand interface around the AN wells. 


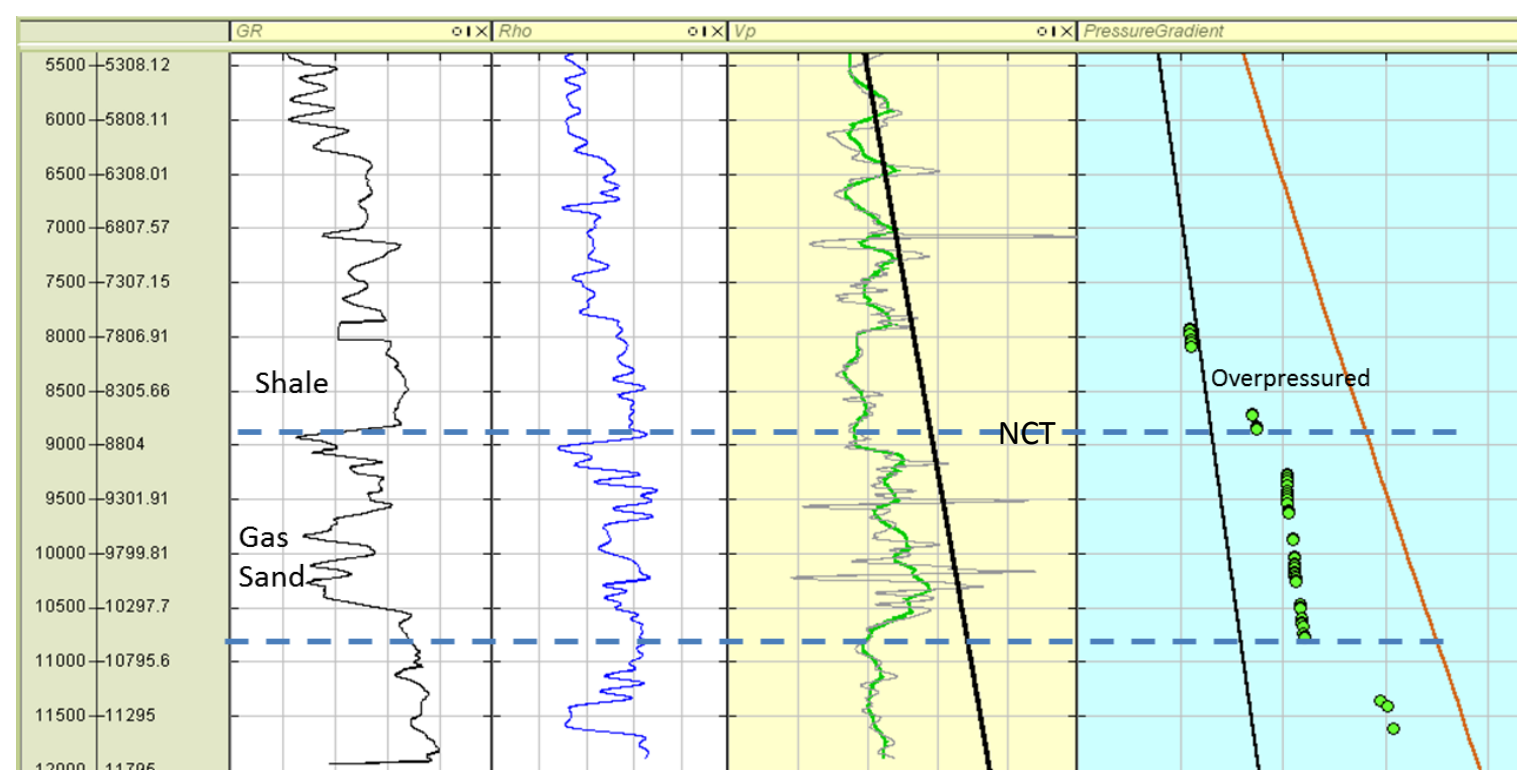

Fig. 6: Log panel views of AN-01 well where overpressure is indicated by P-wave velocity reversal. Little or no change is observed in the shale density with this zone of velocity reversal .NCT is the normal compaction trend.

\section{Conclusion}

Overpressure in the overburden shale accounts for the relatively low P-wave velocity in the shale but not enough to bring the shale density lower than the underlying gas sand. This is due to unloading; a secondary overpressure mechanism. Low frequency bands (i.e. between $15 \mathrm{~Hz}$ and $25 \mathrm{~Hz}$ ) attribute of time-frequency seismic volumes derived from spectral decomposition of full stack depth migration time-frequency seismic volume can give some indications of hydrocarbon gas accumulation in reservoir sands. This can be achieved if mapped reservoir surface attributes at the frequency bands close to the dominant frequency of the seismic are carefully and optimally co-rendered or color blended.

\section{Acknowledgement}

The authors gratefully acknowledge Shell Petroleum Development Company (SPDC) Limited for providing the subsurface data and technical advice as well as permission to publish this work.

\section{References}

[1] Partyka, G., Gridley, J. and Lopez, J., Interpretational Applications of Spectral Decomposition in Reservoir Characterization: The Leading Edge, 18(3), 1999, 353-360

[2] Burnett, M. D. and Castagna, J. P., Advances in Spectral Decomposition and Reflectivity Modeling in the Frio Formation of the Gulf Coast, 2004, Search and Discovery Article 40113

[3] Chen, G., Mattucci, G., Fahmy, B. and Finn, C., Spectral-decomposition response to reservoir fluids from a deepwater West Africa reservoir: GEOPHYSICS, 73 (10), 2008, 23-30,

[4] Castagna, J. P., On the use of seismic data in reservoir characterization: in D. J. O’Meara, (Ed), Reservoir Characterization, (PennWell. 2006)

[5] Sinha, S., Routh, P. S., Anno, P. D., and Castagna, J. P., Spectral decomposition of seismic data with continuous-wavelet transform: Geophysics 70, 2005, 19 - 25

[6] Mallat, S. G., and Zhang, Z., Matching Pursuits with Time-Frequency Dictionaries, IEEE Transaction of Signal Processing, 41, 1993, 3397-3415 